\title{
Blood lipids in patients with high and low physical activity depending on polymorphism of beta-2 and 3-adrenergic receptor genes
}

\author{
Anna Isayeva, Maryna Vovchenko, Anna Shalimova, Valentina Halchynska, Lyudmila Peteneva, \\ Tatiana Bondar
}

Department of Complex Risk Reduction of Chronic Non-Communicable Diseases, Government Institution L.T. Malaya Therapy National Institute of the National Academy of Medical Sciences of Ukraine, Kharkiv, Ukraine

\begin{abstract}
Background. The aim of the study was to investigate the lipid levels in patients with various physical activity and polymorphous variants of ADRB2 (Gln27Glu and Arg16Gly) and ADRB3 (Trp64Arg) genes.

Material and methods. One hundred and fifty subjects were enrolled in the study; mean age was $44.7 \pm 8.9$ y.o. Physical activity was assessed using Omron Walking style III step counter HJ-203-EK pedometer. Lipids were assessed via enzymatic method using Cormay reagent kit (Poland). Genotyping of polymorphous sites of ADRB2 (Gln27Glu, rs1042714 and Arg16Gly, rs1042713) and ADRB3 (Trp64Agr, rs4994) genes was performed using SNP-express-SHOT reagent kits manufactured by Litex Research and Production Company, via real-time polymerase chain reaction.

Results. Individuals walking more than 5000 steps daily with Gly16Gly polymorphism had significantly higher blood lipids. Total cholesterol (TC), triglycerides, LDL and HDL-cholesterol levels were 5.10 (3.86-5.74), 1.13 (0.66-1.51), 3.28 (2.14-3.70), $1.43(1.32-1.63) \mathrm{mmol} / \mathrm{L}$ in Arg16 group and 5.55 (5.23-6.96), 1.56 (0.45$-2.02), 3.87(3.09-4.46)$ and $1.30(1.13-1.68) \mathrm{mmol} / \mathrm{L}$ in Gly16Gly group. The differences in lipid parameters were established for polymorphous variants of $A D R B 3$ gene in patients walking more than 5000 steps daily. In Trp54 carriers, TC, triglycerides, LDL and HDL-cholesterol levels were 5.23 (4.00-5.69), 1.25 (0.80-1.56), 3.25 $(2.22-3.80)$ and $1.41(1.26-1.53) \mathrm{mmol} / \mathrm{L}$, respectively. In 54Arg carriers group, lipids were significantly higher: TC 5.74 (5.59-6.88), triglycerides 1.51 (1.03-1.90), LDL-cholesterol 3.78 (3.38-5.06) and HDL-cholesterol $1.68(1.13-1.81) \mathrm{mmol} / \mathrm{L}$.

Conclusion. This analysis suggests that carriers of Arg 16 of $A D R B 2$ gene and/or Trp54 of ADRB3 gene tend to have lower lipid parameters when they have high physical activity (walking more than 5000 steps a day) in comparison to carriers of 16Gly and/or 54Agr. There is no association between lipids level and ADRB2 (Gln27Glu and Arg16Gly) and ADRB3 (Trp64Agr) polymorphisms when individuals have low physical activity (walking less than 5000 steps a day).
\end{abstract}

Key words: blood lipids; physical activity; $A D R B 2$ and $A D R B 3$ genes polymorphisms

Arterial Hypertens. 2018, vol. 22, no. 4, pages: 193-200

DOI: $10.5603 / A H . a 2018.0020$

\footnotetext{
Address for correspondence: Anna Isayeva

Department of Complex Risk Reduction of Chronic Non-Communicable Diseases, Government Institution L.T. Malaya Therapy National Institute of the National Academy of Medical Sciences of Ukraine, Kharkiv, Ukraine e-mail: anna_isayeva_74@yahoo.co.uk
}

V M Copyright $@ 2018$ Via Medica, ISSN 2449-6170 


\section{Introduction}

Dyslipidaemia is one of the main risk factors of cardiovascular diseases. Polygenic dyslipidaemia along with hypertension and glucose metabolism disturbance is considered as a key compound of metabolic syndrome. European Society of Cardiology (ESC) and European Society of Hypertension (ESH) experts emphasize the necessity of dyslipidaemia treatment and recommend physical activity as an integral part of lifestyle modification.

The onset and progression of polygenic dyslipidaemia is influenced by genetic susceptibility and environmental factors. Beta- 2 and beta- 3 adrenergic receptors play an important role in response to physical exercise, regulate lipid and carbohydrate metabolism. Beta- 2 and beta- 3 adrenergic receptors are encoded by $A D R B 2$ and $A D R B 3$ genes, located on chromosomes 5 and 8 , respectively [1].

Beta-2-adrenergic receptors are involved in fat mobilization from fatty cells for production of energy in response to stimulation via adrenaline and noradrenaline. The presence of amino acid substitutions Gln27Glu and Arg16Gly results in formation of receptor less sensitive to the effect of agonists and causing less pronounced tissue response to stimulation. Stimulation of beta-3-adrenergic receptors results in enhancement of lipolysis. Arg64 carriers have reduced lipolysis level. The presence of the above mutations can modify the body response to physical exercise.

Many candidate genes, polymorphisms of which are associated with the risk of development and severity of dyslipidaemia, have been established. Besides, polymorphous variants of the above genes are related to the body response to physical exercise. The best studied are the loci of the genes $A D R B 2-$ rs 1042714 (531 C>G, Gln27Glu) and rs1042713 (46 A>G, Arg16Gly) and ADRB3 - rs4994 (190T>C, Trp64Arg). According to literature data, different polymorphic forms of $A D R B 2$ are associated with type 2 diabetes mellitus, obesity, nocturnal asthma, and decrease in arterial pressure (at rest, during, and after physical activity) [1]. Concurrently, individuals with genetic alterations in $A D R B 3$ are characterized by lower energy expenditure and basal metabolic rate as compared to individuals without genetic alterations [2].

Physical activity is one of the main elements of lifestyle modification in prevention of lipid metabolism disorders. Meta-analysis by Hanson and Jones showed that group walking promotes the decrease in total cholesterol [3]. The data presented by Mann et al. confirm the beneficial effects of regular activity on cholesterol levels and describe the impacts of different volumes and intensities of exercise on different types of cholesterol [4]. Prusik et al. revealed considerable effect of Nordic walking on lipid metabolism parameters in women [5]. Hagner-Derengowska et al., studying female population, also demonstrated statistically significant effect of walking on blood lipid levels [6]. Williams and Thompson showed that walking and running decrease the risk of development of hypercholesterolaemia in an equally effective manner [7]. The results by Marigliano et al. are in concordance with the data of the above discussed studies and demonstrate considerable positive effect of walking on lipid metabolism parameters [8]. At the same time, meta-analysis presented in 2018 by Oja et al. failed to demonstrate the direct effect of walking on cholesterol, while its essential effect on body mass index (BMI) and blood pressure parameters was proven [9]. An individual's sensitivity to physical exercise as a factor regulating lipid metabolism can be predetermined genetically. That is why the goal of this study was to investigate lipid metabolism parameters associated with different physical activity levels in individuals with polymorphous variants of $A D R B 2$ (Gln27Glu and Arg16Gly) and ADRB3 (Trp64Arg) genes.

\section{Material and methods}

One hundred and fifty subjects were enrolled in the study; median age of the examined individuals was 44.2 [31.1-51.7] years. Thirty-five (23.3\%) study subjects had essential hypertension. Patients with coronary heart disease, proven atherosclerotic lesions of coronary arteries, cardiac failure, patients administered with statins, having disorders of thyroid gland hormone-producing function, suffering from any diseases affecting mobility and physical activity, those on diets with strict limitations of any nutrients, having regular workouts, with body mass index greater than $40 \mathrm{~kg} / \mathrm{m}^{2}$, and suffering from diabetes mellitus type 1 and 2 were not included into the study. Blood pressure-lowering therapy was carried out with ramipril 5-10 mg daily and amlodipine 5-10 mg daily.

\section{Anthropometric parameters}

Measurements of weight, height and waist circumference were performed by trained physician. Body fat, visceral fat and muscular tissue percentage were obtained by bioelectrical impedance using OMRON (Tokyo, Japan) according to the manufacturer's in- 
struction. Body mass index (BMI) was expressed as weight $(\mathrm{kg})$ divided by squared height (meters) $\left(\mathrm{BMI}=\mathrm{kg} / \mathrm{m}^{2}\right)$.

\section{Physical activity}

No additional intervention into the usual lifestyle of examined subjects was performed. The participants' customary physical activity, in particular, walking, was assessed. Physical activity was assessed using Omron Walking style III step counter HJ-203-EK pedometer. The number of steps taken daily was recorded every day during the three consecutive days. Mean number of steps per day was calculated.

\section{Biochemical tests}

Venous blood samples were drawn from each subject after at least 10-h fasting period. Blood lipid spectrum - total cholesterol (TC), high density lipoprotein cholesterol (HDL-cholesterol), triglycerides (TG) were determined via enzymatic method using Cormay reagent kit (Poland). The level of very low density lipoprotein cholesterol (VLDL-cholesterol) was calculated using the formula:

\section{VLDL-cholesterol $=T G / 5$}

The concentration of the LDL-cholesterol was calculated according to Friedwald's formula, i.e.: the difference between total cholesterol and cholesterol concentrations in other lipoprotein fractions:

$L D L$-cholesterol $=T C-(T G / 5+H D L$-cholesterol $)$

\section{Genotyping}

Genotyping of polymorphous sites of ADRB2 (Gln27Glu, $531 \mathrm{C}>\mathrm{G}$, rs1042714 and Arg16Gly, 46 $\mathrm{A}>\mathrm{G}$, rs 1042713) and ADRB3 (Trp64Agr, 190T>C, rs4994) genes was performed using reagent kits SNPexpress-SHOT manufactured by Litex Research and Production Company (Russian Federation), via realtime polymerase chain reaction (PCR). DNA was isolated from whole blood using reagent kit DNA-Sorb-B (Amplisense, Russian Federation).

\section{Statistical analysis}

The normality of variables' distribution was assessed with Kolmogorov-Smirnov test. All data that conformed to normal distribution were expressed as means with SD. Otherwise, the data were presented as medians with corresponding quartiles (Q1; Q3). Statistical significance of differences between groups was assessed using Student's t-test for samples meeting normal distribution criteria, or Mann-Whitney test for samples not meeting normal distribution.

\section{Results}

Baseline characteristics of the study subjects divided into physical activity subgroups (less and more than 5000 steps per day) are presented in Table I. The division point conformed to the mean number of

Table I. Baseline characteristics of the study subgroups formed depending on daily physical activity

\begin{tabular}{|c|c|c|c|c|}
\hline Variable & All $(n=150)$ & $\begin{array}{c}<5000 \text { steps per day } \\
(\mathrm{n}=\mathbf{8 6})\end{array}$ & $\begin{array}{c}>5000 \text { steps per day } \\
(n=64)\end{array}$ & p-value \\
\hline Age (years) & $43.1 \pm 11.2$ & $44.1 \pm 9.9$ & $42.9 \pm 11.1$ & 0.52 \\
\hline Men/women & $64 / 86$ & $40 / 46$ & $24 / 40$ & 0.27 \\
\hline \multicolumn{5}{|l|}{ Anthropometrics } \\
\hline Weight [kg] & $87.2 \pm 23.5$ & $83.5 \pm 28.7$ & $89.9 \pm 25.0$ & 0.17 \\
\hline $\mathrm{BMI}[\mathrm{kg} / \mathrm{m} 2]$ & $30.6 \pm 7.3$ & $31.1 \pm 6.2$ & $28.9 \pm 8.3$ & 0.35 \\
\hline Waist circumference & $95.1 \pm 17.1$ & $92.0 \pm 19.2$ & $94.0 \pm 13.0$ & 0.47 \\
\hline Body fat (\%) & $38.0 \pm 9.6$ & $41.6 \pm 7.4$ & $38.0 \pm 8.7$ & 0.01 \\
\hline Muscle tissue (\%) & $27.2 \pm 5.1$ & $25.2 \pm 4.0$ & $26.1 \pm 5.9$ & 0.28 \\
\hline Visceral fat (\%) & $9.8 \pm 4.7$ & $9.2 \pm 4.4$ & $9.8 \pm 4.1$ & 0.40 \\
\hline \multicolumn{5}{|l|}{ Blood pressure } \\
\hline SBP [mm Hg] & $139.1 \pm 22.7$ & $141.0 \pm 21.7$ & $137.2 \pm 22.1$ & 0.29 \\
\hline $\mathrm{DBP}[\mathrm{mm} \mathrm{Hg}]$ & $84.4 \pm 12.17$ & $80.0 \pm 12.4$ & $82.8 \pm 14.2$ & 0.20 \\
\hline \multicolumn{5}{|l|}{ Lipids } \\
\hline Total cholesterol [mmol/L] & $5.39 \pm 0.97$ & $5.53 \pm 0.91$ & $5.31 \pm 1.07$ & 0.18 \\
\hline Triglycerides [mmol/L] & $1.54 \pm 0.89$ & $1.46 \pm 0.92$ & $1.43 \pm 0.94$ & 0.85 \\
\hline LDL-cholesterol [mmol/L] & $3.39 \pm 0.87$ & $3.23 \pm 0.91$ & $3.39 \pm 0.95$ & 0.30 \\
\hline HDL-cholesterol [mmol/L] & $1.39 \pm 0.39$ & $1.37 \pm 0.39$ & $1.53 \pm 0.42$ & 0.02 \\
\hline
\end{tabular}




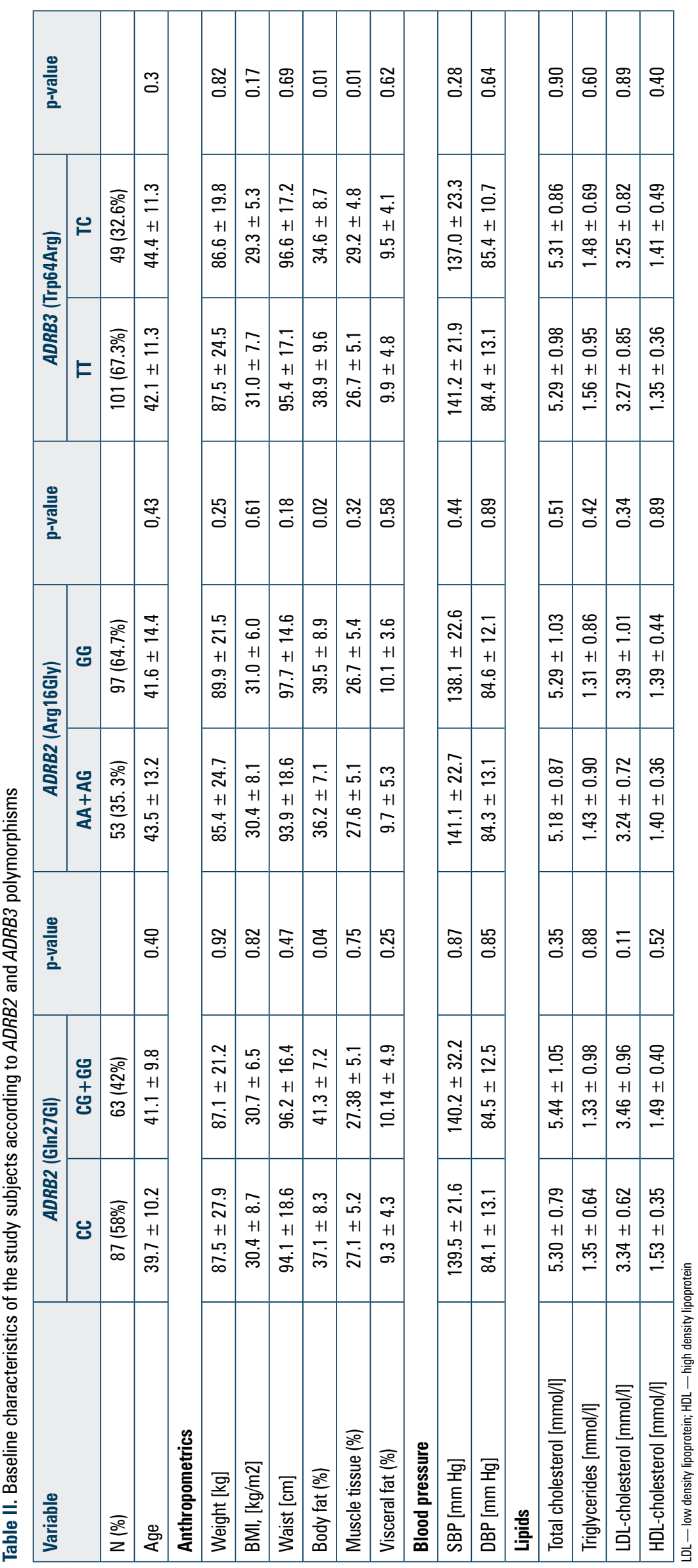


steps in the whole group $(\mathrm{M} \pm \mathrm{SD}, 5051 \pm 1230$ steps per day). Groups with low and high physical activity (of less than 5000 and more than 5000 steps a day, respectively) were comparable in terms of age, sex distribution, blood pressure parameters, and blood lipid parameters. Patients with lower physical activity had higher share of fatty tissue in the body $(p=0.04)$, although there were no significant differences between groups in BMI or shares of muscular tissue and visceral fat. No differences were found in total cholesterol, LDL-cholesterol and triglycerides between groups with high and low physical activity. Individuals with low physical activity had significantly lower HDL-cholesterol ( $\mathrm{p}=0.02)$.

Several models were used for the comparison of polymorphous variants of each studied locus of $A D R B 2$ and $A D R B 3$ genes. Table II presents the characteristics of examined subjects depending on carriage of various alleles and genotypes of polymorphisms of studied genes. Analysis of models CC vs $\mathrm{CG}$ vs $\mathrm{GG}, \mathrm{CC}+\mathrm{CG}$ vs $\mathrm{GG}$ and $\mathrm{C}$ vs $\mathrm{G}$ of $A D R B 2$ gene revealed no significant differences in any of the tested parameters. In model $C \mathrm{C}$ vs $\mathrm{CG}+\mathrm{GG}$, significantly higher fatty tissue content was identified in carriers of CG+GG (27Glu) genotypes.

Analysis of models AA vs AG vs GG, AA vs $\mathrm{AA}+\mathrm{AG}, \mathrm{A}$ vs $\mathrm{G}$ of $A D R B 2$ gene (Arg16Gly) did not reveal significant differences between tested param- eters as well. When the $A A+A G$ vs $G G$ model was used, significantly higher fatty tissue content was established in GG genotype carriers.

Analysis of parameters depending on polymorphous variants of $A D R B 3$ gene established that individuals with TT genotype had higher fatty tissue share and lower muscular tissue share in the body. Body mass index showed no significant differences depending on the carriage of alleles and genotypes of polymorphous variants $A D R B 2$ (Gln27Glu), $A D R B 2$ (Arg16Gly) and $A D R B 3$ (Trp64Arg). Analysis of lipids in subgroups depending on carriage of various polymorphous variants of tested loci failed to identify any significant differences as well.

The comparison of lipid metabolism parameters in carriers of various genotypes with relation to physical exercise is presented in Table III. No considerable differences in lipid levels were identified in the low physical exercise group during assessment of different models.

We carried out a separate analysis of lipid levels in the group of study subjects walking more than 5000 steps daily. Comparison of groups with different genotypes of $A D R B 2$ (Gln27Glu) polymorphism revealed no significant differences during analysis of $\mathrm{CC}$ vs $\mathrm{CG}$ vs $\mathrm{GG}, \mathrm{CC}$ vs $(\mathrm{CC}+\mathrm{CG})$ and $(\mathrm{CC}+\mathrm{CG})$ vs GG models. Assessment of lipid parameters in patient groups with different genotypes of polymor-

Table III. Lipid metabolism parameters in groups with different genotypes of polymorphism of genes ADRB2 (Gln27Glu), ADRB2 (Arg16Gly), ADRB3 (Trp64Arg) in examined subjects with low daily physical activity expressed as $<5000$ steps daily; $\mathrm{n}=86$

\begin{tabular}{|c|c|c|c|}
\hline & \multicolumn{2}{|c|}{ ADRB2 (Gln27Glu) } & \multirow[t]{2}{*}{ p-value } \\
\hline & CC (n= 39) & CG + GG (n= 47) & \\
\hline Total cholesterol [mmol/L] & $5.41[4.88-6.22]$ & $5.70[5.21-6.18]$ & 0.13 \\
\hline Triglycerides [mmol/L] & $1.49[1.02-2.34]$ & $1.46[0.89-2.55]$ & 0.75 \\
\hline LDL-cholesterol [mmol/L] & $2.92[2.79-3.50]$ & $3.40[2.02-4.58]$ & 0.15 \\
\hline \multirow[t]{3}{*}{ HDL-cholesterol [mmol/L] } & $1.40[1.14-1.65]$ & $1.41[1.00-1.78]$ & 0.18 \\
\hline & \multicolumn{2}{|c|}{ ADRB2 (Arg16Gly) } & \multirow[t]{2}{*}{ p-value } \\
\hline & $A A+A G(n=7)$ & GG $(n=59)$ & \\
\hline Total cholesterol [mmol/L] & $5.34[4.74-5.51]$ & $5.37[4.73-6.65]$ & 0.22 \\
\hline Triglycerides [mmol/L] & $1.28[0.83-1.84]$ & $1.33[1.03-1.90]$ & 0.12 \\
\hline LDL-cholesterol [mmol/L] & $3.22[2.81-3.77]$ & $3,78[2,43-4,82]$ & 0.48 \\
\hline \multirow[t]{3}{*}{ HDL-cholesterol [mmol/L] } & $1.37[1.14-1.53]$ & $1.30[0.98-1.55]$ & 0.36 \\
\hline & \multicolumn{2}{|c|}{ ADRB3 (Trp64Arg) } & \multirow[t]{2}{*}{ p-value } \\
\hline & $\mathrm{TT}(\mathrm{n}=64)$ & $\mathrm{TC}(\mathrm{n}=22)$ & \\
\hline Total cholesterol [mmol/L] & $5.37[4.69-6.01]$ & $5.35[5.32-6.59]$ & 0.47 \\
\hline Triglycerides [mmol/L] & $1.35[0.74-1.27]$ & $1.41[0.81-1.70]$ & 0.23 \\
\hline LDL-cholesterol [mmol/L] & 3.17 [2.58-3.69] & $3.15[2.23-4.12]$ & 0.44 \\
\hline HDL-cholesterol [mmol/L] & $1.39[1.07-1.54]$ & $1.28[1.16-1.40]$ & 0.46 \\
\hline
\end{tabular}


Table IV. Lipid metabolism parameters in groups with different genotypes of polymorphism of ADRB2 (Gln27Glu), ADRB2 (Arg6Gly), ADRB3 (Trp64Arg) genes in examined subjects with high daily physical activity expressed as $>5000$ steps daily; $(n=64)$

\begin{tabular}{|c|c|c|c|}
\hline & \multicolumn{2}{|c|}{ ADRB2 (Gln27Glu) } & \multirow[t]{2}{*}{ p-value } \\
\hline & $C C+C G(n=48)$ & $G G(n=16)$ & \\
\hline Total cholesterol [mmol/L] & $5.33[4.00-6.14]$ & $5.31[5.23-5.55]$ & 0.84 \\
\hline Triglycerides [mmol/L] & $1.13[0.88-1.51]$ & $2.33[1.36-3.56]$ & 0.01 \\
\hline LDL-cholesterol [mmol/L] & $3.63[3.20-4.43]$ & $3.09[2.21-3.78]$ & 0.34 \\
\hline HDL-cholesterol [mmol/L] & $1.43[1.30-1.68]$ & 1.13 [1.03-1.53] & 0.35 \\
\hline \multicolumn{4}{|l|}{ ADRB2 (Arg16Gly) } \\
\hline & $A A+A G(n=26)$ & GG $(n=22)$ & \\
\hline Total cholesterol [mmol/L] & $5.10[3.86-5.74]$ & $5.55[5.23-6.96]$ & 0.03 \\
\hline Triglycerides $[\mathrm{mmol} / \mathrm{L}]$ & $1.13[0.66-1.51]$ & $1.56[0.45-2.02]$ & 0.07 \\
\hline LDL-cholesterol [mmol/L] & $3.28[2.14-3.70]$ & $3.87[3.09-4.46]$ & 0.05 \\
\hline HDL-cholesterol [mmol/L] & $1.43[1.32-1.63]$ & $1.30[1.13-1.68]$ & 0.55 \\
\hline \multicolumn{4}{|l|}{ ADRB3 (Trp64Arg) } \\
\hline & $\mathrm{TT}(\mathrm{n}=37)$ & $\mathrm{TC}(\mathrm{n}=27)$ & \\
\hline Total cholesterol [mmol/L] & $5.23[4.00-5.69]$ & $5.74[5.59-6.88]$ & 0.006 \\
\hline Triglycerides [mmol/L] & $1.25[0.80-1.56]$ & $1.51[1.03-1.90]$ & 0.22 \\
\hline LDL-cholesterol [mmol/L] & $3.25[2.22-3.80]$ & $3.78[3.38-5.06]$ & 0.057 \\
\hline HDL-cholesterol [mmol/L] & $1.41[1.26-1.53]$ & $1.68[1.13-1.81]$ & 0.30 \\
\hline
\end{tabular}

LDL — low density lipoprotein; HDL — high density lipoprotein

phism of ADRB2 (Arg16Gly) gene demonstrated that carriers of GG genotype had higher levels of total cholesterol, LDL-cholesterol and HDL-cholesterol, as well as triglycerides, in the high physical activity group compared to carriers of $A A+A G$ genotypes. Lipid parameters also differed depending on $A D R B 3$ (Trp64Arg) polymorphous variants. TC genotype carriers had significantly higher total cholesterol and LDL-cholesterol levels (Table IV).

\section{Discussion}

Our study showed the association between polymorphous variants of $A D R B 2$ (Gln27Glu and Arg16Gly) and $A D R B 3$ (Trp64Arg) and blood lipids level. This association was significant only if individuals have high daily physical activity. Significantly higher total cholesterol levels were identified in carriers of homozygous genotype GG of gene ADRB2 (Arg16Gly) polymorphism and carriers of minor allele $\mathrm{C}$ of gene ADRB3 (Trp64Arg) polymorphism only in a group of patients with higher physical activity.

The obtained results do not allow stating the association of polymorphous variants ADRB2 (Gln27Glu), ADRB2 (Arg16Gly), ADRB3 (Trp64Arg) with cholesterol level in the whole population of examined subjects, while in higher physical activ- ity, association between genetic polymorphisms and lipid metabolism parameters becomes statistically significant.

The above mentioned polymorphous variants are rather widely studied as genetic markers of the risk of obesity, diabetes mellitus, and diet intervention sensitivity disorders [10-13]. Polymorphous locus Trp64Arg of $A D R B 3$ gene was studied in many works [12, 14-16]. Trp64Arg polymorphism decreases sensitivity of beta-3-adrenergic receptor $[17$, 18]. Gene $A D R B 2$ encoding lipolytic receptor in human fatty cells, hepatocytes, muscular cells, associated with lipid mobilization, lipolysis. Arg16Gly and Gln27Glu polymorphisms are deemed the best studied as risk markers of obesity and diabetes mellitus. Change of amino acid sequence on extracellular $\mathrm{N}$-end of $A D R B 2$ in carriers of minor alleles of polymorphisms rs1042713 and rs1042714 can result in $A D R B 2$ dysfunction. Literature describes quite many studies demonstrating the relation between ADRB2 (Gln27Glu), ADRB2 (Arg16Gly) polymorphisms and risk of development, as well as severity of the course of obesity and diabetes mellitus. At the same time, beta-2-adrenergic receptor also plays a role in cholesterol metabolism regulation [19]. The work by Tomaszewski et al. established the association of ADRB2 (Gln27Glu), ADRB2 (Arg16Gly) polymorphisms with cho- 
lesterol levels for Polish patient population with essential hypertension [20]. The authors showed that coexistence of $\operatorname{Arg} 16$ allele and Gln27 allele at the ADRB2 locus showed a borderline association with the increased risk of elevated LDL. Moreover, Arg16-Gln27 ADRB2 configuration, detrimental genotype at neuropeptide Y Leu7Pro doubled the risk of elevated LDL in hypertensive subjects [20]. The fact that these polymorphisms can modulate the lipid metabolism response to external interventions, e.g. dietary ones, is rather interesting. Thus, Ramos-Lopez et al. demonstrated that the decrease in lipid levels in response to dietary interventions would be different in carriers of different genotypes of ADRB2 (Gln27Glu) and ADRB2 (Arg16Gly) polymorphisms [21]. They studied the effects of two types of dietary interventions (moderately high protein program and low fat program) on cholesterol level decrease depending on carriage of ADRB2 (Gln27Glu) and (Arg16Gly) polymorphisms. It should be mentioned that baseline levels of total cholesterol and its fractions did not differ between carriers of different polymorphous gene variants. However, carriers of Gly16Gly genotype ADRB2 (GG) showed less pronounced decrease in total cholesterol and LDL-cholesterol levels when prescribed with moderately high protein program versus the carriers of Arg16 allele (AA+AG). When prescribed with low fat diet, Gly16Gly and Arg16 carriers demonstrated comparable decrease in lipid levels [21]. The study by Ramos-Lopez et al. confirms the fact that polymorphous sites $A D R B 2(\mathrm{Gln} 27 \mathrm{Glu})$ and ADRB2 (Arg16Gly), certainly, do not predetermine the lipid level in general population, but can modulate the response to environmental effects, which in this case was dietary intervention [21].

The role of mutations in ADRB3 gene is also extensively studied. Results of several studies enable concluding that Trp64Arg $(19 \mathrm{~T}>\mathrm{C})$ polymorphism $A D R B 3$ is associated with abnormal function of beta3 adrenergic receptor in the fat tissue [14, 22, 23]. The work by Brondani et al. showed that $\mathrm{C}$ allele (64Arg) of ADRB3 gene was associated with protective effect against obesity. Besides, this work established that carriers of $\mathrm{C}$ allele (64Arg) had higher level of HDL-cholesterol [22]. The study by Chen et al. demonstrated that, in Chinese population, carriers of Trp64Trp genotype of ADRB3 gene had higher triglyceride levels [17]. Our study did reveal the association of TT (Trp64Trp) genotype with higher triglyceride level, which can be explained by interethnic differences.

The goal of our study was not the investigation of relation between the presented polymorphisms and presence of obesity. At the same time, it was established that, although there were no significant differences in BMI between groups with different genotypes of polymorphisms of adrenergic receptor genes, carriers of allele $A(A A+A G)$ genotypes of ADRB2 (Arg16) gene and TT genotype of ADRB3 (Trp64Trp) gene had higher fatty tissue share.

This study did not establish the relation between genotypes of ADRB2 (Gln27Glu) polymorphism and lipid level either in general population of examined subjects or in subgroups with low and high physical activity. At the same time, analysis of lipid parameters in patient groups with different genotypes of polymorphism of ADRB2 gene (Arg16Gly) demonstrated that carriers of homozygous GG genotype (Gly16Gly) with higher physical activity had considerably higher parameters of total cholesterol, triglycerides, and LDL-cholesterol compared to the group of $(A A+A G)$ genotype carriers, which means that these individuals were less sensitive to physical exercise as a factor affecting the lipid level. The second group less sensitive to physical exercise as a factor affecting the lipid metabolism is represented by carriers of minor allele $\mathrm{C}$ of $19 \mathrm{~T}>\mathrm{C}$ polymorphism (rs4994, Trp64Arg) of ADRB3 gene. Thus, the obtained data demonstrate the role of polymorphism carriage in formation of body response to physical exercise. The study conducted by Leońska-Duniec et al. in Polish population also revealed that the response to physical exercise differed depending on carriage of polymorphous variants. Unlike our work, the authors analysed the results of active intervention into patients' lifestyle. It was established during a 12-week aerobic training program that carriers of minor alleles Gly16 and Glu27 of ADRB2 gene demonstrated more pronounced decrease in fatty tissue share [24]. Probably, during lifestyle correction, these patients should take into account their lower sensitivity to aerobic physical exercise. This study has several limitations. The work was conducted in a limited population, $n=150$. Although the physical activity assessment was carried out for several days, only one activity type, walking, was taken into account.

\section{Conclusion}

Carriers of Arg16 (AA+AG) of ADRB2 gene and TT (Trp54) of $A D R B 3$ gene have lower lipid parameters under the conditions of equal physical exercise. Association with polymorphous variants of these genes is manifested only in the case of physical exercise exceeding 5000 steps daily. 


\section{References}

1. Zhang H, Wu J, Yu L. Association of Gln27Glu and Arg16Gly polymorphisms in Beta2-adrenergic receptor gene with obesity susceptibility: a meta-analysis. PLoS One. 2014; 9(6): e100489, doi: 10.1371/ journal.pone.0100489, indexed in Pubmed: 24960039.

2. Naka I, Hikami K, Nakayama K, et al. A functional SNP upstream of the beta- 2 adrenergic receptor gene (ADRB2) is associated with obesity in Oceanic populations. Int J Obes (Lond). 2013; 37(9): 12041210, doi: 10.1038/ijo.2012.206, indexed in Pubmed: 23229733.

3. Baturin AK, Sorokina Elu, Pogozheva AV, et al. [Regional features of obesity-associated gene polymorphism (rs9939609 FTO gene and gene Trp64Arg ADRB3) in Russian population]. Vopr Pitan. 2014; 83(2): 35-41, indexed in Pubmed: 25059067.

4. Pereira AC, Floriano MS, Mota GFA, et al. Beta2 adrenoceptor functional gene variants, obesity, and blood pressure level interactions in the general population. Hypertension. 2003; 42(4): 685-692, doi: 10.1161/01.HYP.0000085648.65419.17, indexed in Pubmed: 12900437.

5. Daghestani $M$, Daghestani $M$, Daghistani $M$, et al. ADRB3 polymorphism rs4994 (Trp64Arg) associates significantly with bodyweight elevation and dyslipidaemias in Saudis but not rs1801253 (Arg389Gly) polymorphism in ARDB1. Lipids Health Dis. 2018; 17(1): 58, doi: 10.1186/s12944-018-0679-7, indexed in Pubmed: 29587766.

6. Maia-Landim A, Ramírez JM, Lancho C, et al. Long-term effects of Garcinia cambogia/Glucomannan on weight loss in people with obesity, PLIN4, FTO and Trp64Arg polymorphisms. BMC Complement Altern Med. 2018; 18(1): 26, doi: 10.1186/s12906-018-2099-7, indexed in Pubmed: 29361938.

7. Szendrei B, González-Lamuño D, Amigo T, et al. PRONAF Study Group. Influence of ADRB2 Gln27Glu and ADRB3 Trp64Arg polymorphisms on body weight and body composition changes after a controlled weight-loss intervention. Appl Physiol Nutr Metab. 2016; 41(3): 307-314, doi: 10.1139/apnm-2015-0425, indexed in Pubmed: 26888112.

8. Chen Y, Wang X, Shen Z, et al. Effect of the beta- 3 adrenergic receptor Trp64Arg and uncoupling protein 1-3826 $\mathrm{A}>\mathrm{G}$ genotypes on lipid and apolipoprotein levels in overweight/obese and non-obese
Chinese subjects. Lipids Health Dis. 2015; 14: 34, doi: 10.1186/ s12944-015-0029-y, indexed in Pubmed: 25928572.

9. Miyaki K, Sutani S, Kikuchi H, et al. Increased risk of obesity resulting from the interaction between high energy intake and the Trp64Arg polymorphism of the beta3-adrenergic receptor gene in healthy Japanese men. J Epidemiol. 2005; 15(6): 203-210, indexed in Pubmed: 16276029.

10. Petrone A, Zavarella S, Iacobellis G, et al. Association of beta 2 adrenergic receptor polymorphisms and related haplotypes with triglyceride and LDL-cholesterol levels. Eur J Hum Genet. 2006; 14(1): 94-100, doi: 10.1038/sj.ejhg.5201521, indexed in Pubmed: 16251889.

11. Tomaszewski M, Charchar FJ, Lacka B, et al. Epistatic interaction between beta2-adrenergic receptor and neuropeptide $Y$ genes influences LDL-cholesterol in hypertension. Hypertension. 2004; 44(5): 689-694, doi: 10.1161/01.HYP.0000143844.81979.61, indexed in Pubmed: 15364898.

12. Ramos-Lopez O, Riezu-Boj JI, Milagro FI, et al. Differential lipid metabolism outcomes associated with ADRB2 gene polymorphisms in response to two dietary interventions in overweight/obese subjects. Nutr Metab Cardiovasc Dis. 2018; 28(2): 165-172, doi: 10.1016/j. numecd.2017.11.006, indexed in Pubmed: 29331538.

13. Brondani LA, Duarte GCK, Canani LH, et al. The presence of at least three alleles of the ADRB3 Trp64Arg (C/T) and UCP1 -3826A/G polymorphisms is associated with protection to overweight/obesity and with higher high-density lipoprotein cholesterol levels in Caucasian-Brazilian patients with type 2 diabetes. Metab Syndr Relat Disord. 2014; 12(1): 16-24, doi: 10.1089/met.2013.0077, indexed in Pubmed: 24138564.

14. Ryuk JAh, Zhang X, Ko BS, et al. Association of $\otimes 3$-adrenergic receptor rs 4994 polymorphisms with the risk of type 2 diabetes: A systematic review and meta-analysis. Diabetes Res Clin Pract. 2017; 129: 86-96, doi: 10.1016/j.diabres.2017.03.034, indexed in Pubmed: 28521197.

15. Leońska-Duniec A, Jastrzębski Z, Jażdżewska A, et al. Individual Responsiveness to Exercise-Induced Fat Loss and Improvement of Metabolic Profile in Young Women is Associated with Polymorphisms of Adrenergic Receptor Genes. J Sports Sci Med. 2018; 17(1): 134-144, indexed in Pubmed: 29535587. 